\title{
Efecto de edulcorantes no calóricos sobre el desarrollo de mermelada de mora (Rubus glaucus Benth)
}

\author{
Eeffect of noncaloric sweeteners on the development of blackberry \\ (Rubus glaucus Benth) jam
}

\author{
Carlos J. Márquez ${ }^{*}$, Birina L. Caballero², Katherin M. Vanegas ${ }^{3}$ \\ Recibido para publicación: Septiembre 15 de 2015 - Aceptado para publicación: Octubre 13 de 2016
}

\begin{abstract}
RESUMEN
Se desarrollaron mermeladas de mora (Rubus glaucus Benth) bajas en calorías, utilizando edulcorantes no calóricos. El trabajo se realizó bajo un diseño experimental de bloques completos al azar con seis tratamientos y cuatro repeticiones. La metodología aplicada fue de acuerdo a la Norma Técnica Colombiana NTC 285. Como control se empleó mermeladas con sacarosa y pectina, los tratamientos fueron mermeladas elaboradas con stevia y sucralosa y agar-agar como gelificante. Se evaluaron $\mathrm{pH}$, acidez, sólidos solubles totales (SST), textura, índice de pardeamiento (IP), características organolépticas y se calculó el aporte calórico para cada tratamiento. Se encontró para el pH de los tratamientos un valor inferior al mínimo establecido por la NTC 285; al igual que la acidez; los SST para el control estuvieron acordes con los recomendados por el CODEX STAN 2962009. Los tratamientos con edulcorantes no calóricos y sus combinaciones presentaron menores SST con respecto al control. En la textura no se evidenció diferencia significativa entre los tratamientos, a partir de sucralosa, stevia y mezclas de estos y el uso de sacarosa como control. El análisis sensorial presentó para los tratamientos sacarosa y stevia (t4), sacarosa y sucralosa (t5) la mayor aceptación, no se encontraron diferencias significativas con respecto al control. La reducción calórica fue de $91,03 \%, 91,02 \%, 90,90 \%$, para los tratamientos stevia (t3), stevia y sucralosa (t6) y sucralosa (t2), respectivamente respecto al control. Se concluye que las mermeladas de mora producidas fueron bajas en calorías y que los tratamientos t4 y t5 fueron los de mayor aceptación.
\end{abstract}

\begin{abstract}
low calorie blackberry jams (Rubus glaucus Benth) were developed using non-caloric sweeteners. An experimental randomized complete block design with 6 treatments and 4 replicates was used. $\mathrm{pH}$, acidity, total soluble solids (TSS), texture, browning index (BI) and organoleptic characteristics were evaluated, the caloric content was calculated for each treatment. The methodology followed the Colombian Technical Standard (NTC 285). Jams with sucrose and pectin served as control, whereas treatments were made with stevia and sucralose and agar - agar as gelling. The $\mathrm{pH}$ of treatments presented a value below the minimum established by the standard NTC 285 and also the acidity. The TSS control were consistent with those recommended by the CODEX STAN 296-2009. For all treatments with non-caloric sweeteners and combinations the TSS were lower compared to the control; the texture presented no significant difference between treatments and control with a confidence level of $95 \%$, sensory analysis for treatments sucrose and stevia (t4) and sucrose and sucralose (t) 5 showed greater acceptance and no significant differences with the control. The caloric reduction was $91.03 \%$, 91.02\%, sucralose $90.90 \%$, for treatments stevia ( $\mathrm{t} 3$ ), stevia and sucralose (t6), and sucralose (t2), respectively, relative to control. The study concluded that the $\mathrm{t} 2, \mathrm{t} 3$ and $\mathrm{t} 6$ treatments are low-calorie products and that $\mathrm{t} 5$ and $\mathrm{t} 4$ were the most widely accepted.
\end{abstract}

Key words: Agroindustry, agar-agar, gels fruit, browning index, pectin.

Palabras clave: Agroindustria, Agar-agar, geles de fruta, índice de pardeamiento, pectin.

\footnotetext{
${ }_{1}^{1}$ Ph.D. Universidad Nacional de Colombia, Sede Medellín. Calle 59a No. 63-20. Medellín, Colombia. Teléfono: 574 4309066, Correo electrónico: cjmarque@unal.edu.co

${ }^{2}$ Ingeniera Agroindustrial. Universidad del Atlántico. Barranquilla, Colombia.

${ }^{3}$ Ingeniera Agrónoma, Universidad Nacional de Colombia, Sede Medellín, Colombia.
} 
Márquez et al. - Mermeladas bajas en calorías

\section{INTRODUCCIÓN}

La mora andina, o mora de Castilla (Rubus glaucus Benth), es nativa de las zonas tropicales de América, principalmente Colombia y Ecuador; actualmente es distribuida a nivel mundial (Finn y Clark 2011; Arozarena et al. 2012). Es una fruta no climatérica, formada por la agregación de los carpelos, como pequeñas drupas insertadas ordenadamente sobre un corazón blando y blanco, de forma cónica, que al madurar fisiológicamente adquiere un color rojo que se torna púrpura con la madurez de consumo, se desarrolla en condiciones óptimas a una altitud entre 1.800 a 2.400 msnm y a una temperatura de $11{ }^{\circ} \mathrm{C}$ a $18{ }^{\circ} \mathrm{C}$ (Jiménez et al. 2009). Posee capacidad antioxidante, atribuida a su contenido de compuestos fenólicos (Ferreira et al. 2010; Denardin et al. 2015) y a la combinación de vitaminas C y E $(21 \mathrm{mg} / 100$ g y 0,71 mg/100 g respectivamente), es rica en pectina, fibra soluble que ayuda a reducir los niveles de colesterol en sangre (Ferreira et al. 2011). Tiene gran aceptación tanto para su consumo en fresco, por su exquisito sabor, aroma y atractivo color, así como por la facilidad para su industrialización como materia prima para la preparación de dulces, jugos, helados, vino y principalmente mermeladas (Kubota et al. 2012; Rios et al. 2014).

Los alimentos gelificados tipo mermelada están definidos por la Norma Técnica Colombiana (NTC 285) como; "Producto pastoso obtenido por la cocción y concentración de pulpa o mezcla de pulpa y jugo de una o más frutas, adecuadamente preparadas con edulcorantes, con la adición o no de agua y de aditivos permitidos". Además la misma norma señala que la concentración final de sólidos solubles totales, por lectura refractométrica, no debe ser inferior al 65\%; generalmente los materiales principales en las mermeladas son; frutas $\mathrm{u}$ hortalizas o sus pulpas, edulcorante normalmente azúcar, agua, agente gelificante y ácido, aunque a veces se utilizan conservantes como sulfito y benzoato de sodio y aditivos como colorantes y aromatizantes, estando su uso condicionado por la normativa del país (Vera 2012). Las frutas ideales en la fabricación de mermeladas deben contener suficiente pectina y ácido, a fin de darle una consistencia de gel sólido o semisólido al producto final (Cámara de Comercio de Bogóta 2015). La NTC 1239 y NTC 3757 establece que un alimento es bajo en calorías cuando presenta una reducción en el contenido calórico de mínimo 35\% con respecto al producto normal correspondiente.

El mercado de productos "light" o bajos en aporte de calorías presenta una tendencia que ha ido aumentando a través de los años entre los consumidores, bien sea por razones estéticas, médicas o porque se buscan productos que contribuyan a una vida más saludable; razón por la cual se investiga sobre el uso de edulcorantes capaces de reemplazar el efecto dulce del azúcar y que aporten menos calorías. Algunos de los edulcorantes utilizados son extractos naturales mientras que otros son sintéticos, para este último caso se denominan edulcorantes artificiales, el empleo de edulcorantes no calóricos como sustitutos de todo o parte del contenido de azúcares en los alimentos, ha tenido su máxima expansión en los últimos 35 años (Anderson et al. 2012). Sin embargo la mayoría de las veces al desarrollar alimentos bajos en calorías, se modifican materias primas presentes en el producto tradicional, como es el caso de la sacarosa que juega un papel importante en la dulzura y textura de los productos en los que participa en su formulación, en especial para las mermeladas (Kmazurkiewicz et al. 2006), además aporta propiedades funcionales a los alimentos, al tener efecto sobre las características sensoriales (sabor), físicas (cristalización, viscosidad), microbianas (preservación, fermentación) y químicas (reacción de maillard, caramelización, antioxidación), entre otras (Brands et al. 2001; Agustí 2010). El objetivo de ésta investigación fue desarrollar productos 
gelificados tipo mermeladas de mora bajas en calorías, utilizando stevia y sucralosa como edulcorantes de bajo aporte calórico.

\section{MATERIALES YMÉTODOS}

Preparación de las muestras. Los procedimientos se realizaron en el Laboratorio de Frutas y Hortalizas de la Universidad Nacional de Colombia Sede Medellín. El material vegetal, fueron moras frescas provenientes de la región rural del municipio de Guarne, departamento de Antioquia - Colombia, en grado de madurez 5 y 6, de acuerdo a la NTC 4106, las frutas fueron previamente lavadas y desinfectadas con hipoclorito de sodio en concentración de 50 ppm, luego se acondicionaron para la obtención de la pulpa. La unidad experimental UE fue de 500 g de pulpa de mora, a los cuales se les midieron los grados brix ${ }^{\circ} \mathrm{Bx}$ y aplicando balance de masas apropiado se calculó la cantidad de sacarosa necesaria, pectina y agua evaporada para obtener una mermelada con $68{ }^{\circ} \mathrm{Bx}$ finales de concentración. A partir del poder edulcorante de la stevia:sacarosa 1:250 y la sucralosa:sacarosa 1:600 se calcularon las cantidades de los edulcorantes a adicionar para los demás tratamientos y el porcentaje de sólidos solubles totales \%SST final para los tratamientos elaborados con edulcorantes no calóricos el cual fue de 11,6 ${ }^{\circ} \mathrm{Bx}$ luego de la evaporación y los ${ }^{\circ} \mathrm{Bx}$ finales de las combinaciones con la sacarosa fueron de $53{ }^{\circ} \mathrm{Bx}$. Para el control el agente gelificante fue pectina cítrica rápida

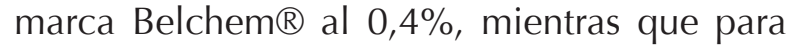
los tratamientos con edulcorantes no calóricos se usó como agente gelificante agar-agar marca

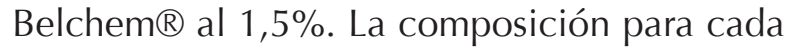
tratamiento se muestra en la tabla 1.

Análisis físico-químicos. Para la caracterización fisicoquímica de las mermeladas, se evaluaron, los sólidos solubles totales en porcentaje ( $g$ SST/g fase líquida $x$ 100) se determinaron con el método refractométrico utilizando un refractómetro Leica auto $\mathrm{ABBE}$ con escala de 0-82\% y se expresó en ${ }^{\circ} \mathrm{Bx}$, el $\mathrm{pH}$ se determinó con un potenciómetro Schott CG840B y la acidez por titulación potenciométrica con $\mathrm{NaOH} 0,1 \mathrm{~N}$ y se expresó como (\% de ácido cítrico). La medida del color de la mermelada se determinó utilizando el espectrofotómetro X-RITE, modelo SP60, con un iluminante D65 y un ángulo del observador de $10^{\circ}$ con una apertura de visión de $8 \mathrm{~mm}$, se obtuvieron las coordenadas de color CIE-L*a*b*, donde $\mathrm{L}^{*}$ es el indicador de luminosidad, $\mathrm{a}^{*}$ indica cromaticidad en el eje verde(-) a rojo (+), y b* sobre el eje azul (-) a amarillo (+), con las cuales se calculó el Índice de Pardeamiento (IP) para cada una de las UE, utilizando las Ecuaciones 1 y 2 (Mascan 2001).

$$
I P=\frac{10(\mathrm{x}-0,31)}{0,172} \quad \text { Ecuación } 1
$$

Donde: $\quad X=\frac{\left(a^{*}+1,75 L^{*}\right)}{\left(5,645 L^{*}+a^{*}-3,012 b^{*}\right)} \quad$ Ecuación 2

Tabla 1. Composición porcentual de las mermeladas de mora (Rubus glaucus Benth) con base en los edulcorantes calóricos y no calóricos.

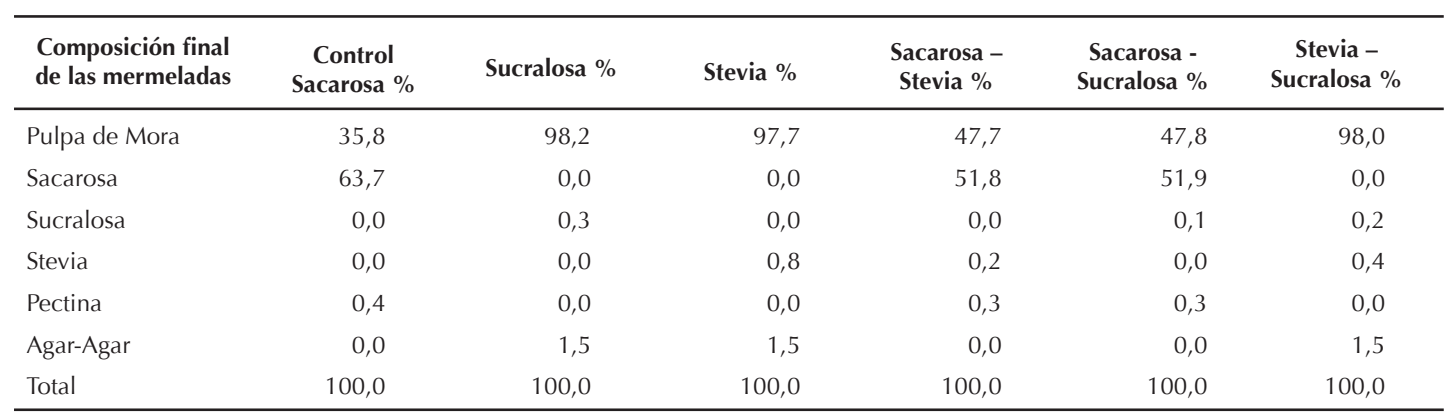


La firmeza del gel se determinó utilizando un analizador de textura TA-XT2i (Stable Micro Systems) y el Software Texture Expert Excced, Versión 2,64, se midió con un cono 45 Conical Perpex, con un diámetro de 29,97 mm y una altura de 38,21 mm; la velocidad de penetración fue de $2 \mathrm{~mm} \mathrm{~s}-1$ con un recorrido de la sonda de $10 \mathrm{~cm}$, como unidad de medida se utilizó el Newton (N). El cálculo calórico (Cal) se determinó de acuerdo a la composición final de cada tratamiento expresado por contenido en cada recipiente de vidrio de $325 \mathrm{~g}$.

Análisis sensorial. Los atributos sensoriales fueron evaluados por 15 jueces conocedores de las características propias de una mermelada de mora, aplicando una prueba de diferencia crítica entre las sumas de los rangos comparando cada uno de los tratamientos con respecto al control, con un nivel de confianza del 95\%, estableciendo que la diferencia crítica se presenta para diferencias entre los tratamientos con valores mayores o iguales a 30, la prueba consistió en ordenar las muestras según su nivel de aceptación general de 1 a 6 , en una escala donde, 1 es la calificación de la mermelada de mayor aceptación y 6 corresponde a la calificación de la mermelada de menor aceptación para cada uno de los jueces (Watts et al. 1992).

Diseño experimental y análisis estadístico. Se realizó un diseño de bloques completos al azar con seis tratamientos y cuatro repeticiones para un total de 24 UE, a las cuales se les evaluaron las características físico-químicas; SST, pH, acidez, IP, textura y calorías, se aplicaron análisis descriptivos y pruebas de Anova con un nivel de confianza del 95\%. Se utilizó el paquete estadístico Statgraphics Centurion XV.II.

\section{RESULTADOS Y DISCUSIÓN}

Los análisis de las características físicoquímicas para cada una de las diferentes formulaciones de mermeladas se presentan en la tabla 2, donde el porcentaje de los SST de las formulaciones de mermeladas elaboradas con edulcorantes no calóricos y en combinación con la sacarosa t2, t3, t4, t5 y t6, obtuvieron valores inferiores a lo establecido por la NTC 285 en comparación con t1, (véase Tabla 2) esto debido a que al utilizar stevia y sucralosa, o su combinación, como edulcorantes no calóricos, estos no aportan carbohidratos y por lo tanto es notoria la disminución de calorías en el alimento.

Es importante establecer que en las formulaciones con edulcorantes no calóricos se retiró por evaporación directa la cantidad de masa de agua equivalente a la mermelada control o t1.

Con respecto al $\mathrm{pH}$ los tratamientos difieren en comparación con lo establecido por la

Tabla 2. Características fisicoquímicas de las mermeladas de mora (Rubus glaucus Benth).

\begin{tabular}{|c|c|c|c|c|c|c|}
\hline Tratamientos & $\%$ SST $\left({ }^{\circ} \mathbf{B x}\right)$ & $\mathrm{pH}$ & Acidez (\%) & Textura (N) & $\begin{array}{c}\text { Índice de } \\
\text { Pardeamiento (IP) }\end{array}$ & $\begin{array}{l}\text { Calorías } \\
\text { por } 325 \mathrm{~g}\end{array}$ \\
\hline t1 & $68,25 \pm 2,36 a$ & $2,57 \pm 0,10 a$ & $2,46 \pm 0,75 a$ & $13,61 \pm 1,43 a$ & $8,53 \pm 4,90 a$ & $828,72 \mathrm{a}$ \\
\hline t2 & $8,75 \pm 0,95 b$ & $2,75 \pm 0,08 b$ & $4,50 \pm 0,74 b$ & $7,99 \pm 2,15 b$ & $51,89 \pm 13,88 b$ & $74,63 \mathrm{~b}$ \\
\hline t3 & $9,25 \pm 0,95 b$ & $2,76 \pm 0,08 b$ & $4,99 \pm 1,78 b$ & $12,0 \pm 5,08 a b$ & $50,73 \pm 9,79 b$ & $74,26 \mathrm{~b}$ \\
\hline t4 & $54,25 \pm 6,70 \mathrm{c}$ & $2,72 \pm 0,09 \mathrm{c}$ & $2,52 \pm 0,25 \mathrm{a}$ & $17,6 \pm 2,90 \mathrm{c}$ & $12,17 \pm 8,87 \mathrm{c}$ & $687,50 \mathrm{c}$ \\
\hline t5 & $51,5 \pm 4,20 \mathrm{c}$ & $2,72 \pm 0,07 \mathrm{c}$ & $2,98 \pm 0,46 a$ & $12,7 \pm 6,01 a b$ & $12,15 \pm 6,06 \mathrm{c}$ & $688,31 \mathrm{c}$ \\
\hline t6 & $8,5 \pm 2,81 b$ & $2,80 \pm 0,06 \mathrm{c}$ & $4,63 \pm 1,10 b$ & $13,26 \pm 5,31 \mathrm{ab}$ & $56,61 \pm 11,11 b$ & $74,44 \mathrm{~b}$ \\
\hline
\end{tabular}

Los valores muestran la media y el error estándar para $n=4$. Letras iguales no presentan diferencia significativa para los valores de las columnas.

Donde: t1 Sacarosa; t2 Sucralosa; t3 Stevia; t4 Sacarosa y Stevia; t5 Sacarora y Sucralosa; y t6 Stevia y Sucralosa 
norma NTC 285 para mermeladas elaboradas con frutas ácidas entre ellas la mora, con un mínimo de 3,0 y el máximo de 3,4, encontrándose los tratamientos en un intervalo inferior al mínimo establecido con un valor de 2,7; para el porcentaje de acidez expresado como (ácido cítrico), el mínimo establecido por la norma NTC 285 es de 0,5\%, sin embargo se encontraron para los tratamientos valores superiores, con intervalo entre $2,4 \%$ a $4,9 \%$. Estos valores obtenidos se deben probablemente a las características fenotípicas particulares de éste fruto, puesto que la pulpa de mora en fresco con la que se trabajó presentaba 6 ${ }^{\circ} \mathrm{Bx}$, un $\mathrm{pH}$ de 2,78 $\pm 0,64$ y un porcentaje de acidez de $3,1 \pm 0,57$, valores que se encuentran en el límite inferior de madurez para la mora (NTC 4106). La mayor cantidad de ácido cítrico puede ser debida a la concentración relativa debido a la evaporación, no obstante estos ácidos son importantes para ayudar a prolongar la vida útil, conferir mejor brillo al producto final y mejorar el sabor en contraste con la dulzura (Coronado y Hilario 2001).

La diferencia presentada en el IP de las formulaciones que contenían sacarosa con respecto a las elaboradas con edulcorantes no calóricos, se debe probablemente a que la coloración generada por el pardeamiento debido al tratamiento térmico depende de la concentración de pulpa que aporta los sustratos propios para la reacción de pardeamiento no enzimático, principalmente azúcares reductores, por tanto aquellas formulaciones con mayor contenido de sacarosa fueron las de menor IP con respecto a los tratamientos que fueron formulados con edulcorantes no calóricos (Manayay y Ibarz 2010).

La figura 1 presenta el análisis de la textura, para las seis formulaciones de mermeladas, se encontró que no hay diferencia significativa entre las medias de los tratamientos, t1, t3, t5 y t6, lo cual quiere decir que el agar- agar utilizado como agente gelificante en las mermeladas bajas en calorías presentó una firmeza similar a la pectina cítrica, utilizada en la elaboración de las mermeladas control, y que se usa comúnmente en los productos tradicionales.

La pectina cítrica es el agente gelificante más comúnmente empleado en la elaboración de mermeladas, le da consistencia y textura de gel al producto (Devia 2003), sin embargo, éste valor de textura se debe a que la rigidez del gel depende de la relación alta de azúcar y

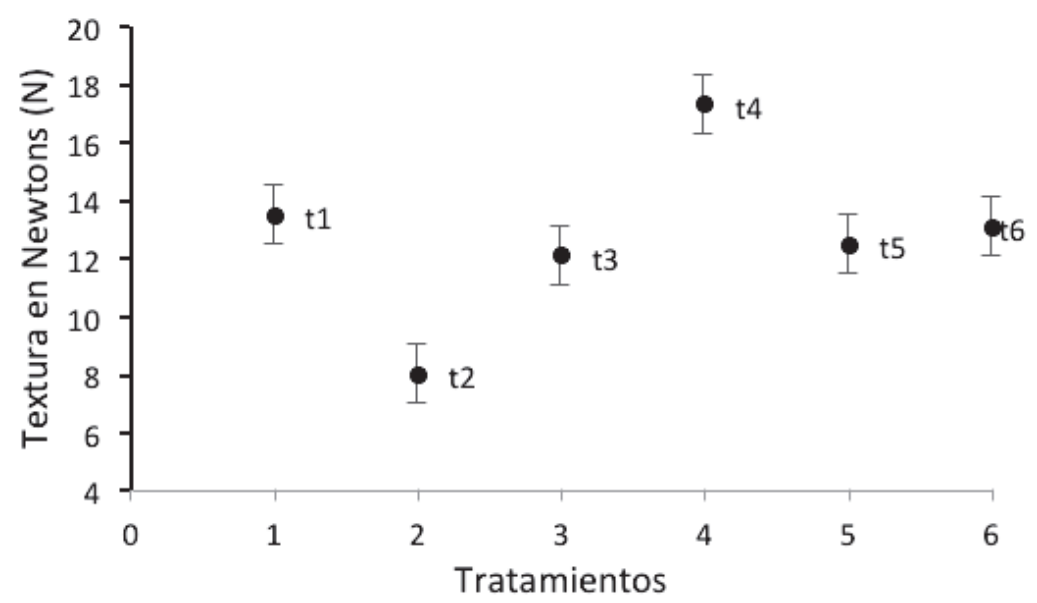

Figura 1. Valor medio para la textura de las mermeladas de mora (Rubus glaucus Benth), los símbolos representan la media para cada tratamiento y las barras verticales el error estándar para $\mathrm{n}=6$. 
ácido, normalmente se tienen concentraciones de azúcar mayores al $60 \%$ y de ácido mayor al 0,5\%. Una alta concentración de azúcar hace que sea mayor la cantidad de agua atrapada por la red cristalina, mientras una alta concentración de ácido aumenta la dureza del gel, pero un exceso puede generar hidrólisis de la pectina; bajas concentraciones de ácido producen fibras tan blandas que la estructura del gel sería incapaz de soportar el líquido y se formarían grumos indeseables (Garrido et al. 2015).

El otro agente gelificante utilizado para los tratamientos bajos en calorías correspondientes a los tratamientos t2, t3 y t6, fue el agar-agar en concentración de 1,5\%, el cual es una mezcla de polisacáridos obtenidos de algas rojas conocidas como agarófitas y está compuesto por heteropolisacáridos formado por moléculas de $\beta$-D-galactosa, 3,6-anhidro- $\alpha$-L-galactosa, que le confiere alta fuerza al gel, en un rango de temperatura entre $32{ }^{\circ} \mathrm{C}$ y $39^{\circ} \mathrm{C}$, dándole textura a las formulaciones de dulces y productos gelificados (Fennema 2000; Maldonado y Singh 2008). Además, el agar-agar es utilizado como base de solidificación en diferentes matrices, con muchas otras aplicaciones en las industrias de alimentos, médica, biotecnológica y farmacéutica, en ésta investigación los geles desarrollados con agar-agar presentaron una resistencia a la penetración del gel similar a los formulados y desarrollados con pectina cítrica (Freile et al. 2002).

La determinación del aporte calórico de cada tratamiento, fue expresada en calorías por recipiente de $325 \mathrm{~g}$, teniendo en cuenta que el control aportó 828,7 Cal; para que un producto se considere bajo en calorías, debe tener un porcentaje de $35 \%$ menos de calorías que la mermelada tradicional o control formulada con sacarosa, lo anterior de acuerdo a la NTC 1239 y NTC 3757, por lo que las mermeladas bajas en calorías deberían tener un aporte de 538,7 Cal o menos por los 325 g de producto final; se observó que para los tratamientos t4 y t5 hubo una reducción en calorías del 17,04\% y 16,94\% respectivamente, mientras que los tratamientos t3, t6 y t2 presentaron una reducción en calorías del $91,03 \%, 91,02 \%$ y $90,9 \%$ respectivamente, por lo tanto con ese porcentaje de disminución estas formulaciones podrían ser consideradas como mermeladas bajas en calorías.

De acuerdo a la evaluación sensorial presentada en la tabla 3, el tratamiento con el mayor grado de aceptación fue $t 1$, mientras que t2 fue el de menor aceptación.

Tabla 3. Orden de preferencia de los jueces para las mermeladas desarrolladas con edulcorantes calóricos y no calóricos.

\begin{tabular}{ccc}
\hline $\begin{array}{c}\text { Orden de preferencia } \\
\text { de los jueces }\end{array}$ & Tratamientos & Calificación \\
\hline $\mathbf{1}$ & $\mathrm{t} 1$ & 22 \\
$\mathbf{2}$ & $\mathrm{t} 4$ & 30 \\
$\mathbf{3}$ & $\mathrm{t} 5$ & 45 \\
$\mathbf{4}$ & $\mathrm{t} 6$ & 63 \\
$\mathbf{5}$ & $\mathrm{t} 3$ & 75 \\
$\mathbf{6}$ & $\mathrm{t} 2$ & 80 \\
\hline
\end{tabular}

Orden de preferencia por ordenación (Watts et al. 1992).

Donde: 11 Sacarosa; t2 Sucralosa; t3 Stevia; t4 Sacarosa y Stevia; t5 Sacarora y Sucralosa; y t6 Stevia y Sucralosa.

En la tabla 4 se puede apreciar como los tratamientos t4 y t5 no presentaron diferencias críticas con respecto al control con un nivel de confianza del 95\%, debido a que sus diferencias en la sumatoria son menores al valor crítico establecido para 6 tratamientos y 15 jueces el cual es de 30, por lo tanto, podría decirse que cualquiera de estos tratamientos puede reemplazar a la mermelada tradicional en cuanto a los aspectos sensoriales (Watts et al. 1992).

Los tratamientos $\mathrm{t} 2$, $\mathrm{t} 3$ y $\mathrm{t} 6$ presentaron un valor superior a la diferencia crítica de 30 por lo tanto su calidad sensorial no reemplazaría al control. 
Tabla 4. Comparación de diferencias críticas de suma de rangos entre los tratamientos y el control en el análisis sensorial.

\begin{tabular}{cc}
\hline $\begin{array}{c}\text { Diferencias para el control } \\
\mathrm{y} \text { los tratamientos }\end{array}$ & $\begin{array}{c}\text { Diferencia crítica } \\
\text { entre las sumas de rangos }\end{array}$ \\
\hline $\mathrm{t} 1-\mathrm{t} 4$ & 8 \\
$\mathrm{t} 1-\mathrm{t} 5$ & 23 \\
$\mathrm{t} 1-\mathrm{t} 6$ & $41^{*}$ \\
$\mathrm{t} 1-\mathrm{t} 3$ & $53^{*}$ \\
$\mathrm{t} 1-\mathrm{t} 2$ & $58^{*}$ \\
\hline
\end{tabular}

*Para 15 jueces y seis tratamientos la diferencia crítica entre las sumas de rangos se establece para valores $\geq 30$ (Watts et al. 1992). Donde: t1 Sacarosa; t2 Sucralosa; t3 Stevia; t4 Sacarosa y Stevia; t5 Sacarora y Sucralosa; y t6 Stevia y Sucralosa.

\section{CONCLUSIONES}

Los tratamientos formulados con stevia (t3), stevia y sucralosa (t6), y sucralosa (t2) presentaron una reducción en calorías del $91,03 \%, 91,02 \%$ y $90,90 \%$ con respecto al control, por lo tanto pueden ser considerados como productos bajos en calorías.

La evaluación sensorial de las mermeladas elaboradas con la combinación de edulcorantes sacarosa y stevia (t4), sacarosa y sucralosa (t5), no presentaron diferencias estadísticamente significativas con respecto al control (t1).

El menor índice de pardeamiento (IP) lo presentaron los tratamientos con presencia de sacarosa (t1), (t5) y (t4) respectivamente.

El agar-agar puede ser usado como agente gelificante para la elaboración de mermeladas bajas en calorías en reemplazo de la pectina cítrica.

\section{AGRADECIMIENTOS}

Los autores expresan su agradecimiento al personal de los Laboratorios de Frutas y Hortalizas y de Control de Calidad de Alimentos de la Universidad Nacional de Colombia sede Medellín, especialmente a María Isabel Betancur Nieto y Fernando Arenas Gil.

\section{REFERENCIAS}

Agustí, R. 2010. Influencia de los tratamientos térmicos en la elaboración de productos untables de tomate formulados con isomatulosa-frutosa o sacarosa. Tesis de Máster universitario en Gestión y Seguridad Alimentaria. Universidad Politecnica de Valencia, España.

Anderson, G., Foreyt, J., Sigman, M., and Allison, D. 2012. The use of low-calorie sweeteners by adults: impact on weight management. The Journal of nutrition 142(6): 1163s-1169s.

Arozarena, I., Ortiz, J., Hermosín-Gutiérrez, I., Urretavizcaya, I., Salvatierra, S. and Córdova, I. 2012. Color, ellagitannins, anthocyanins, and antioxidant activity of Andean blackberry (Rubus glaucus Benth.) wines. Journal of Agricultural and Food Chemistry 60(30): 7463-7473.

Brands, C. and Van, M. 2001. Reactions of monosaccharides during heating of sugar-casein Systms: buiding of a reaction networt model. Journal of Agricultural and Food Chemistry 49(10): 4667-4675.

Cámara de Comercio de Bogóta. 2015. Manual mermelada: Programa de apoyo agrícola y agroindustrial. Vicepresidencia de fortalecimiento empresarial, Bogotá, p11-14.

Coronado, M. e Hilario, R. 2001. Elaboración de mermeladas. Centro de investigación, educación y desarrollo, Lima, p5-11.

Denardin, C., Hirsch, G., Da Rocha, R., Vizzotto, M., Henriques, A., Moreira, J. and Emanuelli, T. 2015. Antioxidant capacity and bioactive compounds of four Brazilian native fruits. Journal of Food and Drug Analysis 23(3): 387-398.

Devia, J. 2003. Proceso para producir pectinas cítricas. Revista Universidad EAFIT 129: 21-30.

Fennema, O. 2000. Química de los Alimentos. Acribia, Zaragoza, p220-265. 
Márquez et al. - Mermeladas bajas en calorías

Ferreira, D., Rosso, V. y Mercadante, A. 2010. Compostos bioativos presentes em amorapreta (Rubus spp.). Revista Brasileira de Fruticultura 32(3): 664-674

Ferreira, P., Da Silva, V., Rodrigues, A., Gevehr, C., Silva, J., y Da Silva, R. 2011. Benefits of blackberry nectar (Rubus spp.) relative to hypercholesterolemia and lipid peroxidation. Nutrición Hospitalaria: Organo oficial de la Sociedad Española de Nutrición Parenteral y Enteral 26(5): 984-990.

Finn, C. and Clark, J. 2011. Emergence of blackberry as a World crop. Chronica Horticulturae 51(3): 13-18.

Freile, Y., Robledo, D., Pedersén, M., Bruno, E. y Rönnqvist, J. 2002. Effect of dark and salinity treatment in the yield and quality of agar from Gracilaria cornea (Rhodophyceae). Ciencias Marinas 28(3): 289-296.

Garrido, J., Lozano, J., y Genovese, D. 2015. Effect of formulation variables on rheology, texture, colour, and acceptability of apple jelly: Modelling and optimization. LWT - Food Science and Technology 62(1): 325-332.

ICONTEC (El Instituto Colombiano de Normas Técnicas y Certificación). 1996. Arequipe o dulce de leche. ICONTEC (NTC 3757), 9p.

ICONTEC. 1997. Frutas frescas - mora de castilla (Rubus glaucus Benth). ICONTEC (NTC 4106), 13p.

ICONTEC. 2002. Helados y mezclas de helados. ICONTEC (NTC 1239), 18p.

ICONTEC. 2007. Frutas procesadas, mermeladas y jalea de frutas. ICONTEC (NTC 285), 11p.

Jiménez, D., Cock, J., Satizábal, H., Barreto S., Pérez, A., Jarvis, A. And Damme, P. 2009. Analysis of andean blackberry (Rubus glaucus) production models obtained by means of artificial neural networks exploiting information collected by small-scale growers in Colombia and publicly available meteorological data. Computers and Electronics in Agriculture 69(2): 198-208.
Kmazurkiewicz, J., Rebilas, K. And Tomasik, P. 2006. Dextran-low-molecular saccharide sweetener interactions in aqueous solutions. Food Hydrocolloids January 20(1): 21-23.

Kubota, M., Ishikawa, C., Sugiyama, Y., Fukumoto, S., Miyagi, T. And Kumazawa, S. 2012. Anthocyanins from the fruits of rubus croceacanthus and rubus sieboldii, wild berry plants from Okinawa, Japan. Journal of Food Composition and Analysis 28(2): 179-182.

Maldonado, S. y Singh, C. 2008. Efecto de gelificantes en la formulación de dulce de yacón. Ciência $\mathrm{E}$ Tecnologia de Alimentos 28(2): 429-434.

Manayay, D. e Ibarz, A. 2010. Modelamiento de la cinética de reacciones del pardeamiento no enzimático y el comportamiento reológico, en el proceso térmico de jugos y pulpas de fruta. Scientia Agropecuaria 1(2):155-168.

Mascan, M. 2001. Kinetic of colour of kiwi fruits during hot air and microwave drying. Journal of Food Engineering 48(2): 169-175.

OMS (Organización Mundial de la Salud) y FAO (Organización de las Naciones Unidas para la alimentación y la agricultura). 2009. Norma del Codex para la confitura, jaleas y mermeladas. (CODEX STAN 296), 10p.

Rios, V., Pimienta, P., Marques, A., Oliveira, L., Pio, R. And Queiroz, F. 2014. Analysis of the subtropical blackberry cultivar potential in jelly processing. Journal of Food Science 79(9): 1776s-1781s.

Vera, M. 2012. Elaboración de mermelada light de durazno. Tesis en Ingeniero en Alimentos. Universidad de Chile, Santiango de Chile.

Watts, B., Ylimaki, G., y Jeffery, L. 1992. Métodos sensoriales básicos para la evaluación de alimentos. Centro Internacional de Investigaciones para el Desarrollo (CIID), Ottawa, p70-85, p132. 